\title{
MOBILE CROWD SENSING FOR URBAN COMPUTING
}

\author{
Prajakta Joglekar ${ }^{1}$ and Vrushali Kulkarni ${ }^{2}$
}

\begin{abstract}
Urban computing aims to improve the quality of life of ordinary citizens by providing sustainable solutions. This paper reviewed how Mobile Crowd Sensing can be used for urban computing. The paper listed the features and advantages of mobile crowd sensing mechanism. It highlighted the challenges involved in mobile crowd sensing. The paper also presented a comprehensive study of Apps used to monitor ambient noise data, and compared the ways in which the listed challenges have been tackled in those Apps. The authors proposed a framework for better aggregation analysis of crowdsensed data, which would help in event discovery of urban region.
\end{abstract}

Keywords - urban computing; mobile crowd sensing; MCS; citizen science; noise pollution; event detection

\section{INTRODUCTION}

Urban computing is an interesting research area which has gained momentum with the concept of Smart Cities. According to the Population Reference Bureau, it is expected that $70 \%$ of the world's population would be urban by 2050 . Such an increase in population density in urban areas demands adequate provision of infrastructure, services and technology to meet the needs of the people [1]. The pillars of any modern city include environment, healthcare, transport, education, economy and security. Needless to say, a city forms a complex, social ecosystem, and urban computing and management becomes a challenging area.

So, what is urban computing? According to [2], urban computing involves data acquisition, integration and analysis to create smart environment and improve the quality of life of its citizens. Improving the quality of life includes urban planning, solving traffic problems, reducing pollution and energy conservation. The citizens are the generators as well as the consumers of data. This data can be used to build a sustainable framework for urban management.

Urban data is big data characterized by high volume, high velocity and heterogeneity. There are multiple sources of data and many ways to capture such data. Examples of data sources include GPS data, traffic data, call detail record from mobile phone signals, environmental monitoring data, energy consumption data as well as social network data. With the advent of IoT, lot of devices emit data at continuous rate. Sensors are deployed at various location amount to stream data, which lack computational capability at source. Human as a sensor is an emerging paradigm, where people contribute data by posting text and images on social media, leaving GPS traces or capturing environmental information using smart phone sensors. The smart phone has sensing,

${ }^{1}$ Department of Computer Engineering Maharashtra Institute of Technology, Pune, Maharashtra, India

${ }^{2}$ Department of Computer Engineering Maharashtra Institute of Technology, Pune, Maharashtra, India 
computing as well as communication facility [3]. This paper delves more into how humans can be used as sensors, particularly focusing on the concept of Mobile Crowd Sensing.

The remainder of the paper is as follows: Section II introduces readers to the concept of Mobile Crowd Sensing (MCS), its features and advantages. Section III talks about challenges in MCS. Section IV gives a feature list of Smart Phone Apps which can be used to capture Noise Data, and states how these Apps have tackled various challenges. Section V states the observations from the survey done in the earlier section. It proposes a general framework for event discovery with mobile crowdsensed data using Big Data Architecture. The paper then concludes with scope for future work.

\section{MOBILE CROWDSENSING}

Mobile crowd sensing is based on a distributed problem solving model [4], in which ordinary citizens can contribute the data sensed or generated from their mobile devices. According to the latest statistics from Google, the number of mobile phone users in the world would expectedly cross the 5 billion mark by 2019. Every such user can participate in data capture using MCS method. This data is then aggregated on a central server and intelligent data mining is performed to provide smart solutions for resilient urban framework. In their work [4], the authors have done a comprehensive review of MCS paradigm, by listing its features, presenting applications enabled by MCS and proposing a conceptual framework for such systems. They have also discussed the challenges and research opportunities for mobile crowd sensing. Our work takes inspiration from their work, however we focus more on sensing environmental data and literature related to noise level monitoring using MCS paradigm.

Mobile crowd sensing involves crowd sourcing as well as participatory sensing mechanisms. One can capture user data (e.g. location), ambient data (e.g. noise levels) as well as social data (e.g social events) using MCS technique. Smart phones have inbuilt sensors (e.g 3D accelerometers, microphones, gyroscopes, magnetometers, compass, GPS, light etc). Along with high computational power and emerging 4G wireless standards, Mobile Crowd Sensing paradigm looks to be a promising method of data capture [5].

Users can perform myriads of activities on their smart phones, amounting to huge amount of heterogeneous data being generated. The activities include online shopping, social networking, audio/video streaming, GPS traces, using sensors to measure ambient temperature, noise etc. Users can choose to monitor the events or instruct mobiles to send data automatically to the server. The former concept is called participatory sensing and the latter one is called opportunistic sensing. MCS offers high granularity in space and time, as the user can readily go to the desired place at desired time to capture the data. This is not possible in case of sensors which are deployed at fixed locations.

MCS is essentially a digital form of citizen science. Citizen science enables researcher to collect data on a spatio-temporal scale [6], which, otherwise, is not possible without great investment of time and cost.

\section{A. $\quad$ Features and advantages of Mobile Crowdsensing}

1. Increases citizen's awareness towards his ambience and society.

2. Deployment cost is low, as compared to sensors in WSN, as the user already possesses a phone. In other words, MCS leverages existing sensing and communication infrastructure along with the mobility feature of mobile user.

3. Every area is accessible for capturing data as the phone is a very portable device. Thus, MCS provides fine spatial granularity. 
4. Data can be captured in both, obtrusive as well as unobtrusive ways, saving on user's time.

5. Other than just the environment readings, smart phones can also capture users' feelings or remarks regarding the readings. E.g. Apps like NoiseTube enable the user to sense noise levels as well as attach tags describing the situation, like party music, or traffic jam.

6. Such data can be aggregated and sent to the municipal corporation, where officers can understand the urban dynamics and improve on town planning. It also helps the authority and police to take immediate action in case of disaster or untoward incident.

7. Mobile Apps can be built with disruption tolerant services so that data is captured and stored locally first, and then transmitted whenever network connection is available.

\section{Challenges IN MOBILE CROWDSENSING}

Though all the advantages mentioned in the above section makes MCS look promising, there are many challenges faced by MCS applications. These challenges include data quality, inconsistency, sparsity problem, privacy issues, resource limitations, aggregation issues and incentive mechanisms [3 and 4].

1. Data Quality

As the citizens themselves are the owners of their device, they can intelligently contribute high quality data. This is a boon as well as a disadvantage. Humans may capture wrong data or collect data from a wrong place. Humans may experiment with sensors and send false, garbage data to the server. Smartphone sensors themselves are prone to error too. So, data quality is one of the primary concerns in mobile crowdsensing.

\section{Data redundancy and inconsistency}

Along with quality of data, redundancy and inconsistency pose challenge in MCS systems. E.g. if, while measuring ambient noise levels, 2 users standing at same location record noise levels on their phones and upload it on the server, this is a case of data redundancy. Now, if these 2 readings don't match with each other due to calibration problem or background noise, it's an inconsistency issue.

\section{Data sparsity problem}

If we employ enough users to ensure that the required target area is covered, data sparsity problem would not arise. This involves cost in terms of energy consumption, network bandwidth and incentive mechanisms, which the users may not readily agree to bear with. To solve this problem, the authors of [7] have introduced a Sparse MCS framework to achieve satisfactory data quality.

\section{Resource Limitations}

As there are multiple sensors within single smart phone device, identifying and scheduling of sensing and communication tasks becomes a challenge. GPS data is high quality data, but it drains phone battery faster. Also, different applications may want to use GPS data at the same time, thus requiring efficient resource allocation mechanisms. Same problem exists with bandwidth. How different crowd sensing applications utilize the same bandwidth is a challenge for this paradigm.

\section{Privacy issues}

While on one hand, GPS enabled applications can help solve traffic congestion problem at a city level, on the other hand they tend to invade individual's privacy. Such applications collect sensitive data like the current location of the individual, the daily commuting route etc. 


\section{Incentive mechanisms}

Human involvement in MCS calls for appropriate incentive mechanisms. Participants have to put in explicit or implicit efforts to sense and capture the data. Without strong incentives, humans may not participate willingly in such applications. It is a challenge to keep the users engaged while capturing the measurements on the phone. Some of the incentives which attract users include: interesting task (gamification), enjoyment, service, altruism, financial rewards, ranking and social recognition etc.

\section{Aggregation issues}

Effective decisions can be taken by urban authorities, if quality data from smartphones is sent to the central server and aggregated to produce heat maps and reports, visualizing the pulse of a city. This can also be used to build predictive models to improve the resilience of the city. However, this requires efficient data mining algorithms on the server. Sometimes, real time data needs to be analyzed and acted upon using data stream mining algorithms.

\section{SURVEY OF URBAN NOISE RELATED MCS APPLICATIONS}

In order to understand how the above challenges are tackled in smart phone applications, we selected 'Urban noise computing' as area of focus. Noise pollution is as harmful as air and water pollution, impacting health and wellbeing of citizens. Continuous exposure to loud noise may result in hearing loss, high blood pressure, psychological disorder or change in social behaviour of the people. Hence, it is necessary to capture ambient noise levels to know our daily exposure to noise, and take steps to avoid continuous exposure to loud noise. Citizens' participation is the best way of collecting noise data, as they can immediately sense it on their smart phones and send it to the city officers if the noise is intolerable.

We have done a survey of some literature papers describing mobile crowdsensing applications related to noise pollution data. Table 1 lists such applications, describing how various challenges related to mobile crowd sensing have been handled while capturing noise levels and generating noise maps. This is not a comprehensive list, however, it gives an idea of the overall picture.

Table -1 Smartphone Apps capturing urban noise levels using crowdsensing method

\begin{tabular}{|l|l|l|l|l|}
\hline $\begin{array}{l}\text { S. } \\
\text { No. }\end{array}$ & Application & Description & $\begin{array}{l}\text { Challenges } \\
\text { handled }\end{array}$ & $\begin{array}{l}\text { Methods to handle } \\
\text { challenges }\end{array}$ \\
\hline $\mathbf{1}$ & NoiseTube [8] & $\begin{array}{l}\text { Using this App, citizens can use } \\
\text { their mobile phones to capture } \\
\text { urban noise in DB(A), tag and } \\
\text { upload tit to visualize noise maps }\end{array}$ & $\begin{array}{l}\text { Incentive; Privacy } \\
\text { locial translucence, } \\
\text { generated and her email id } \\
\text { is not displayed. }\end{array}$ \\
\hline $\mathbf{2}$ & $\begin{array}{l}\text { NoizCrowd } \\
\text { [9] }\end{array}$ & $\begin{array}{l}\text { A crowd-based Data Gathering } \\
\text { and Management System for } \\
\text { Noise Level Data }\end{array}$ & $\begin{array}{l}\text { Aggregation } \\
\text { analysis; Data } \\
\text { sparsity }\end{array}$ & $\begin{array}{l}\text { Use of SciDB for data } \\
\text { intensive calculations; k- } \\
\text { nearest neighbour } \\
\text { interpolation (Manhantten } \\
\text { 3distance) for spatial } \\
\text { interpolation }\end{array}$ \\
\hline $\mathbf{3}$ & $\begin{array}{l}\text { Ear-Phone [10 } \\
\text { and 11] }\end{array}$ & $\begin{array}{l}\text { This App does a context aware } \\
\text { noise mapping using smart }\end{array}$ & $\begin{array}{l}\text { Resource } \\
\text { limitations; Data }\end{array}$ & $\begin{array}{l}\text { Context classifier using } \\
\text { accelerometer data and kNN } \\
\text { algorithm to start sensing }\end{array}$ \\
\hline
\end{tabular}




\begin{tabular}{|c|c|c|c|c|}
\hline & & phones. & sparsity & $\begin{array}{l}\text { only when phone is outdoor } \\
\text { and held in hand, thereby } \\
\text { saving energy and } \text { CPU } \\
\text { utilization; 11- norm } \\
\text { minimization } \\
\text { interpolation }\end{array}$ \\
\hline 4 & $\begin{array}{l}\text { NoiseMap } \\
\text { [12] }\end{array}$ & $\begin{array}{l}\text { Mobile crowd sensing based } \\
\text { system to gather noise } \\
\text { measurements and propose noise } \\
\text { abatement measures to city } \\
\text { managers. }\end{array}$ & $\begin{array}{l}\text { Data Sparsity; } \\
\text { Incentive; } \\
\text { Privacy; } \\
\text { Aggregation } \\
\text { Analysis }\end{array}$ & $\begin{array}{l}\text { Decision tree classifier } \\
(\mathrm{C} 4.5) \text { to predict noise } \\
\text { levels from other datasets } \\
\text { like OSM; Open data } \\
\text { access, ranking, ; choice to } \\
\text { keep measurements private; } \\
\text { DBScan Clustering } \\
\text { algorithm }\end{array}$ \\
\hline 5 & $\begin{array}{l}\text { NoiseSPY } \\
{[13]}\end{array}$ & $\begin{array}{l}\text { Using this App, the users can } \\
\text { explore the city area and collect } \\
\text { and visualize noise levels real } \\
\text { time. }\end{array}$ & $\begin{array}{l}\text { Resource } \\
\text { limitations }\end{array}$ & $\begin{array}{l}\text { Logging options are given } \\
\text { to the user to save data } \\
\text { space. }\end{array}$ \\
\hline 6 & $\begin{array}{l}\text { SoundOfTheC } \\
\text { ity [14] }\end{array}$ & $\begin{array}{l}\text { This project uses an unobstrusive } \\
\text { method to capture noise levels, } \\
\text { upload and aggregate it centrally } \\
\text { to produce noise visualization } \\
\text { map }\end{array}$ & $\begin{array}{l}\text { Resource } \\
\text { limitations }\end{array}$ & $\begin{array}{l}\text { The App doesn't send noise } \\
\text { data to the server if the } \\
\text { phone is in the pocket or } \\
\text { indoor. }\end{array}$ \\
\hline 7 & Zappatore [5] & $\begin{array}{l}\text { Data captured is aggregated, } \\
\text { filtered and interpolated on } \\
\text { Hadoop to provide city managers } \\
\text { with noise pollution levels along } \\
\text { with noise abatement measures }\end{array}$ & $\begin{array}{l}\text { Privacy; } \\
\text { Aggregation } \\
\text { Analysis }\end{array}$ & $\begin{array}{l}\text { Owner information and } \\
\text { metadata is not sent to the } \\
\text { server, the mobile devices } \\
\text { are indexed to their IMEI } \\
\text { code (International Mobile } \\
\text { Equipment Identity); HDFS } \\
\text { based Apache Hive for ETL } \\
\text { and aggregation of data }\end{array}$ \\
\hline 8 & $\begin{array}{l}\text { NoiseBattle, } \\
\text { NoiseQuest } \\
{[15]}\end{array}$ & $\begin{array}{l}\text { This App uses gamification } \\
\text { techniques to engage users in } \\
\text { capturing data }\end{array}$ & $\begin{array}{l}\text { Incentive; Data } \\
\text { Quality }\end{array}$ & $\begin{array}{l}\text { Gamification }- \text { capture } \\
\text { higher noise levels to score } \\
\text { more; Consider time of the } \\
\text { day and location factors for } \\
\text { data quality }\end{array}$ \\
\hline
\end{tabular}

\section{V.OBSERVATION}

We observe that data sparsity problem has been considered in most Apps and interpolation methods have been applied. Many of the Apps also offer various incentives to engage users. Privacy preservation is an area of challenge in MCS. Some works like PEPSI architecture [16] 
and Virtual Wall concept [17] have delved in this research area to come up with innovation solutions.

We further observe that though the problem of aggregation analysis has been addressed by few Apps, the application at the server only generates noise maps or displays area wise average noise levels. Few of the applications predict noise levels using machine learning algorithms. However, not much work has been done on event discovery in urban areas using noise data. In his survey paper [2], Zheng has mentioned many good papers where city events or anomalies are detected, however most of them rely on taxi trajectory data, check-in data or 311 complaint data. Such datasets are very difficult to obtain as open datasets in Indian context. We would therefore like to look into this area of research to find out whether interesting events can be detected using measured noise data. To this effect, we envisage a big data framework with machine learning support to store and process MCS data.

We propose an outline framework in this paper, to support MCS mechanism and machine learning using Big Data platform. The framework is shown in Figure 1.The framework consists of following stages:

1. Data capture stage - Data is captured by citizens using mobile crowd sensing. E.g. Noise levels

2. Data pre-processing - Data uploaded on the servers by different smart phones, should be pre-processed to find out noisy data, missing attributes or duplicate records. Normalization should be done for contextual attributes if required. Unwanted features should be removed

3. Storage and Processing on Apache Spark - Apache Spark is powerful Big Data processing Engine with Machine Learning support in the form of MLib. It uses resilient distributed datasets (RDDs) for fast computing and aggregation. Since the mobile crowd sensed data will be huge in volume a city level, it can be stored using Spark into HDFS.

4. Event discovery - A suitable classification, clustering or anomaly detection algorithm can be applied to discover various events in the city using MCS data.

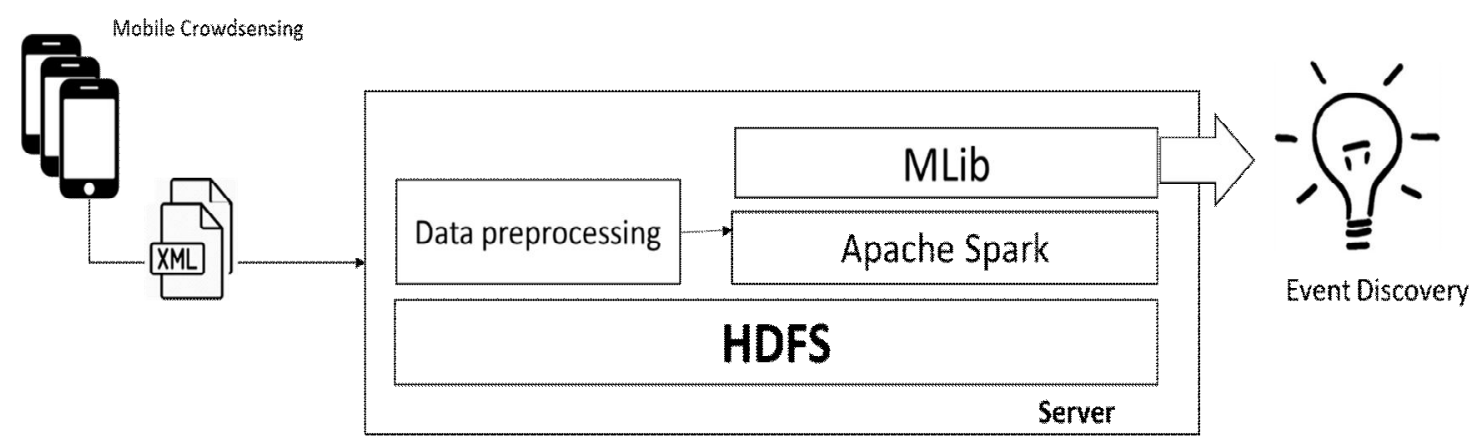

Figure 1. A general framework for urban event discovery using MCS data

We believe that this framework would leverage the power of ordinary citizens in sensing data and such data when mined efficiently, can help the city planners understand urban dynamics and discover new events and concepts in the city. Event detection is a step towards making the city smart. 


\section{CONCLUSION AND FUTURE WORK}

In this paper, we saw how Mobile Crowd Sensing paradigm leverages the power of ordinary citizens to collect huge amount of data in urban computing. Mobile crowd sensing comes with its own set of advantages and challenges. Different techniques have been employed to overcome these challenges in numerous applications.

The data captured using MCS needs efficient mining algorithms in order to extract interesting patterns and events related to urban city life. The framework proposed by us would help in transition of raw data captured using MCS into valuable knowledge, which would help understand the urban dynamics. To the best of our knowledge, no work has yet been done to detect events in the city using noise data measured by MCS method. In future, we propose to design and implement an efficient algorithm to detect interesting events in the city by combining urban noise computing with mobile crowd sensing.

\section{REFERENCES}

[1] Jin et. al., "An Information Framework for Creating a Smart City Through Internet of Things", IEEE Internet of Things Journal ( Volume: 1, Issue: 2), 2014

[2] Zheng et. al., "Urban Computing: Concepts, Methodologies, and Applications", ACM Transactions on Intelligent Systems and Technology, Vol. 5, No. 3, Article 38, 2014

[3] Ganti et. al., " Mobile Crowdsensing: Current State and Future Challenges", IEEE Communications Magazine ( Volume: 49, Issue: 11), 2011

[4] Guo et. al., "Mobile Crowd Sensing and Computing: The Review of an Emerging HumanPowered Sensing Paradigm”, ACM Computing Surveys, Vol. 48, No. 1, Article 7, 2015

[5] Zappatore et. al., "Improving Urban Noise Monitoring Opportunities via Mobile CrowdSensing", Book - Smart City 360 , Springer International Publishing, isbn - 978-3-31933681-7, 2016

[6] D'Hondt et. al., "Participatory noise mapping works! An evaluation of participatory sensing as an alternative to standard techniques for enviromental monitoring", In Proceedings of the 9th International Conference on Pervasive Computing. Springer, 33-36, 2011

[7] Wang et. al., "Sparse Mobile Crowdsensing: Challenges and Opportunities", IEEE Communications Magazine August 2016

[8] I.N. Athanasiadis et. al., "NoiseTube: Measuring and mapping noise pollution with mobile phones", Information Technologies in Environmental Engineering, Environmental Science and Engineering, Springer, 2009

[9] Wisniewski et. al., "NoizCrowd: A Crowd-Based Data Gathering and Management System for Noise Level Data", 10th International Conference, MobiWIS 2013, Paphos, Cyprus, August 26-29, 2013.

[10] Rana et. al., "Ear-phone: A context-aware noise mapping using smart phones", In eprint arXiv:1310.4270, 2013

[11] Rana et. al., "Ear-phone: An end-to-end participatory urban noise mapping system", In Proceedings of the 9th ACM/IEEE International Conference on Information Processing in Sensor Networks. ACM, 105-116, 2014 
[12] Schweizer et. al., "NoiseMap - Realtime participatory noise maps", Second International Workshop on Sensing Applications on Mobile Phones, 2011

[13] Kanjo, "NoiseSPY: A Real-Time Mobile Phone Platform for Urban Noise Monitoring and Mapping”, 15: 562. doi:10.1007/s11036-009-0217-y, 2010

[14] Ruge et. al., "SoundOfTheCity - Continuous Noise Monitoring for a Healthy City", Pervasive Computing and Communications Workshops (PERCOM Workshops), IEEE International Conference, 2013

[15] Marti et. al., "Mobile Application for Noise Pollution Monitoring through Gamification Techniques", Entertainment Computing - ICEC 2012 Volume 7522 of the series Lecture Notes in Computer Science pp 562-571, 2012

[16] Cristofaro and Soriente, "Participatory Privacy: Enabling Privacy in Participatory Sensing", IEEE Network ( Volume: 27, Issue: 1, January-February 2013 )

[17] Kapadia et. al., "Virtual Walls: Protecting Digital Privacy in Pervasive Environments", Pervasive Computing Volume 4480 of the series Lecture Notes in Computer Science pp $162-179,2007$ 